\title{
Complexity Measures for Quantifying Changes in Electroencephalogram in Alzheimer's Disease
}

\author{
Ali H. Husseen Al-Nuaimi (iD, Emmanuel Jammeh (D), \\ Lingfen Sun (D), and Emmanuel Ifeachor
}

Signal Processing and Multimedia Communication (SPMC) Research Group, Faculty of Science and Engineering, School of Computing, Electronics, and Mathematics, University of Plymouth, Plymouth, UK

Correspondence should be addressed to Ali H. Husseen Al-Nuaimi; ali.al-nuaimi@plymouth.ac.uk

Received 15 September 2017; Revised 26 December 2017; Accepted 4 February 2018; Published 13 March 2018

Academic Editor: Hugo Leonardo Rufiner

Copyright (C) 2018 Ali H. Husseen Al-Nuaimi et al. This is an open access article distributed under the Creative Commons Attribution License, which permits unrestricted use, distribution, and reproduction in any medium, provided the original work is properly cited.

\begin{abstract}
Alzheimer's disease $(\mathrm{AD})$ is a progressive disorder that affects cognitive brain functions and starts many years before its clinical manifestations. A biomarker that provides a quantitative measure of changes in the brain due to AD in the early stages would be useful for early diagnosis of $\mathrm{AD}$, but this would involve dealing with large numbers of people because up to $50 \%$ of dementia sufferers do not receive formal diagnosis. Thus, there is a need for accurate, low-cost, and easy to use biomarkers that could be used to detect AD in its early stages. Potentially, electroencephalogram (EEG) based biomarkers can play a vital role in early diagnosis of $\mathrm{AD}$ as they can fulfill these needs. This is a cross-sectional study that aims to demonstrate the usefulness of EEG complexity measures in early $\mathrm{AD}$ diagnosis. We have focused on the three complexity methods which have shown the greatest promise in the detection of AD, Tsallis entropy (TsEn), Higuchi Fractal Dimension (HFD), and Lempel-Ziv complexity (LZC) methods. Unlike previous approaches, in this study, the complexity measures are derived from EEG frequency bands (instead of the entire EEG) as EEG activities have significant association with $\mathrm{AD}$ and this has led to enhanced performance. The results show that $\mathrm{AD}$ patients have significantly lower TsEn, HFD, and LZC values for specific EEG frequency bands and for specific EEG channels and that this information can be used to detect AD with a sensitivity and specificity of more than $90 \%$.
\end{abstract}

\section{Introduction}

Alzheimer's disease (AD) is an age-related progressive, neurodegenerative disorder that is characterized by loss of memory and cognitive decline $[1,2]$ and it is the main cause of disability among older people [3]. AD is ranked as the sixth leading cause of death in US [4]. The rapid increase in the number of people living with $\mathrm{AD}$ and other forms of dementia due to the ageing population represents a major challenge to health and social care systems worldwide [5]. Currently, there are over 46.8 million individuals with dementia worldwide with an annual cost of care estimated at US $\$ 818$ billion and is projected to reach 74.7 million by 2030 with an annual cost of US\$2 trillion [6]. The number of individuals with dementia worldwide is expected to exceed 131 million by 2050 which will have a huge economic impact [7]. However, many dementia sufferers do not receive early diagnosis $[7,8]$. It is estimated that up to $50 \%$ of people living with dementia may not have received formal diagnosis $[8,9]$. In 2011, 28 million people of 36 million dementia sufferers did not receive a diagnosis worldwide [10].

Degeneration of brain cells due to AD starts many years before the clinical manifestations become clear [5, 11-15]. An early diagnosis of $\mathrm{AD}$ will contribute to the development of effective treatments that could slow, stop, or prevent significant cognitive decline [16-18]. An early diagnosis of $\mathrm{AD}$ could also be useful for identifying dementia sufferers who have not received a formal early diagnosis and this may provide an opportunity for them to access appropriate health care services [19-21].

A biomarker that can measure degeneration of brain cells due to $\mathrm{AD}$ at an early stage would be useful for its early diagnosis $[2,22-24]$. But this may require dealing with large numbers of people as up to $50 \%$ of people living with 
dementia may not have received a formal diagnosis. Therefore, there is a need for simple, noninvasive, low-cost, and reliable biomarkers for early diagnosis which can be accessed in clinical practice $[5,25,26]$. Recent guidelines promote the use of biochemical and neuroimaging biomarkers to improve the diagnosis of AD. Cerebral spinal fluid (CSF) testing for $\mathrm{AD}$ is not widely used in clinical practice because it requires lumbar puncture which is an invasive procedure [2, 27, 28]. Neuroimaging is expensive, available only in specialist centres [29], and may not be suitable for patients that have pacemakers or certain implants [30]. Blood-based biomarkers have shown promising results in $\mathrm{AD}$ diagnosis but they are not yet fully developed and low-cost biosensors to detect $\mathrm{AD}$ biomarkers in blood do not exist at present [2, 25, 31].

Potentially, the electroencephalogram (EEG) can play a valuable role in the early diagnosis of $\mathrm{AD}[11,20,21,24$, 32-34]. EEG is noninvasive, low-cost, has a high temporal resolution, and provides valuable information about brain dynamics in $\operatorname{AD}[20,21,33,35,36]$. The fundamental utility of EEG to detect brain signal changes even in the preclinical stage of the disease has been demonstrated $[33,37,38]$. Thus, EEG biomarkers may be used as a first-line decision-support tool in AD diagnosis $[11,35]$ and could complement other AD biomarkers [26].

$\mathrm{AD}$ is characterized by loss of memory and cognitive decline resulting from damage to brain cells which influence brain activity [38]. AD causes changes in the features of the EEG $[35,38,39]$ and EEG analysis may provide valuable information about brain dynamics due to $\mathrm{AD}[20,21,33,35]$. The most characteristic features in EEG caused by AD are slowing of EEG, a decrease in EEG coherence, and reduction in EEG complexity [33-35, 37, 38, 40,41]. These changes in the EEG can be quantified as a biomarker of AD. A variety of linear and nonlinear methods are being developed to quantify changes in EEG as AD biomarkers [42, 43]. AD biomarkers based on the slowing in EEG and a decrease in EEG coherence are often derived using linear analysis methods (i.e., spectral analysis of the EEG signal) [37, 44, 45], while biomarkers extracted by analysing the complexity of the EEG are based on nonlinear approaches (e.g., entropy methods, fractal dimension, and Lempel-Ziv complexity). The EEG complexity approaches have shown promising results in $\mathrm{AD}$ diagnosis $[11,35,46]$ and appear to be appropriate for $\mathrm{AD}$ diagnosis [38, 47, 48]. Complexity is a measure of the extent to which the dynamic behavior of a given sequence resembles a random one [49]. The cortical areas of the brain fire spontaneously and this dynamic behavior of the brain is complex $[50,51]$. AD causes a reduction in neuronal activity of the brain [52] resulting in decreased capability of the brain to process information [53-55] and this may be reflected in the EEG signals [52]. EEG complexity can potentially be a good biomarker for AD diagnosis [38] as AD patients have a significant reduction in EEG complexity [38, 40, 41, 52, 56, 57]. Several studies have investigated EEG complexity as a potential AD biomarker using whole EEG record with the objective of achieving a high performance. Given the association of EEG activities (e.g., alpha, delta activities) with $\mathrm{AD}$, we hypothesized that the derivation of EEG complexity based on EEG activities should lead to enhanced performance.
This is a cross-sectional study aimed at demonstrating the usefulness of EEG based complexity measures to detect AD. In this study, we investigated an important class of complexity measures, information theoretic methods, which offers a potentially powerful approach for quantifying changes in the EEG due to $\mathrm{AD}$ [58]. Information theoretic methods (i.e., TsEn and LZC) have emerged as a potentially useful complexity-based approach to derive robust EEG biomarkers of $\mathrm{AD}[47,58-62]$. They are attractive because of the potential natural link between information theory-based biomarkers and changes in the brain caused by AD [58]. Conceptually, information processing activities in the brain are thought to be reflected in the information content of the EEG.

In particular, TsEn approach has been shown to be one of the most promising information theoretic methods for quantifying changes in the EEG $[62,63]$. It has also been shown to be a reliable analysis tool to use with working memory tasks. As its computation is fast, it can serve as a basis for a real-time decision-support tool for dementia diagnosis by both specialists and nonspecialists [64]. Sneddon et al. [65] investigated TsEn of the EEG and was able to detect mild dementia due to $\mathrm{AD}$ with a sensitivity of $88 \%$ and specificity of $94 \%$. De Bock et al. [62] found TsEn of the EEG to be a highly promising potential diagnostic tool for mild cognitive impairment (MCI) and early dementia with a sensitivity and specificity of $82 \%$ and $73 \%$, respectively. Using TsEn approach, Al-Nuaimi et al. [35] detected AD from normal subjects with a sensitivity and specificity of $85.8 \%$ and $70.9 \%$, respectively. Garn et al. [66] investigated the use of TsEn to diagnose AD based on EEG analysis and achieved a $p$ value $<0.0036$ for channels T7 and T8 in discriminating between $\mathrm{AD}$ patients and normal subjects.

LZC is a nonparametric, nonlinear measure of complexity for finite length sequences [67]. It is a simple and powerful method which has been used in several biomedical applications [68]. LZC depends on a coarse-grain processing of the measurements [69] and can be applied directly on physiologic signal without preprocessing [70]. LZC has been applied extensively in analysing biomedical signals (e.g., EEG) to measure the complexity of discrete-time physiologic signals [67]. Furthermore, it is used to analyse brain function, brain information transmission, and EEG complexity in patients with $\mathrm{AD}$ [43]. The LZC approach produces a good biomarker for $\mathrm{AD}$ detection [70, 71]. Hornero et al. [72] used LZC to analyse EEG and magnetoencephalogram (MEG) in AD patients. They found that LZC provides a good insight into the EEG background activity characteristics and the changes associated with AD. Hornero et al. [73] found that LZC values were lower in $\mathrm{AD}$ patients and suggested that the most relevant differences are in the posterior region. In addition, they suggested that the MEG activity from $\mathrm{AD}$ patients is characterized by a lower degree of irregularity and complexity and that the LZC measures can be used to detect AD with a sensitivity and specificity values of $65 \%$ and $76.2 \%$, respectively. McBride et al. [56] analysed EEG complexity based on the LZC method to discriminate between patients with early MCI, AD patients, and normal subjects. They found that EEG complexity features for specific EEG frequency bands with regional electrical activity provide promising results 
in discriminating between $\mathrm{MCI}, \mathrm{AD}$, and normal subjects. Fernandez et al. [74] analysed MEG complexity for MCI patients, $\mathrm{AD}$ patients, and normal subjects based on LZC method for discriminating between the three groups. They found that a combination of age and posterior LZC scores allowed them to distinguish between $\mathrm{AD}$ patients and MCI patients with $94.4 \%$ sensitivity and specificity.

HFD is a fast computational method for obtaining the fractal dimension of time series signals [75-77] even when very few data points are available [75]. It can track changes in a biosignal from a measure of its complexity [75, 76] and it is suited to capturing region-specific neural changes due to $\mathrm{AD}[45,77]$. In addition, HFD provides a more accurate measure of the complexity of signals compared to other methods $[75,78,79]$ and it has been shown to be an efficient method for discriminating between $\mathrm{AD}$ patients and normal subjects [31, 80]. HFD of the EEG is potentially a good biomarker of $\mathrm{AD}$ diagnosis as it is significantly lower in $\mathrm{AD}$ patients than in normal subjects [46, 80, 81]. Smits et al. found that HFD is sensitive to neural changes selectively related to $\mathrm{AD}$ patients and normal subjects. Al-Nuaimi et al. [46] investigated HFD of EEG for AD diagnosis and they found that HFD is a promising EEG biomarker that captures changes in the regions of the brain thought to be affected first by $\mathrm{AD}$ and it could be used to detect $\mathrm{AD}$ with sensitivity and specificity values of $100 \%$ and $80 \%$, respectively.

It is widely accepted that $\mathrm{AD}$ causes a decrease in the power of high frequencies (alpha, beta, and gamma) and an increase in the power of low frequencies (delta and theta) $[11,33,34,38,41]$. We hypothesized that complexity measures based on the EEG frequency bands would provide better results than those derived directly from the whole EEG record. The aim was to enhance the performance of the complexity measures and to demonstrate their usefulness in quantifying changes in EEG due to $\mathrm{AD}$.

Digital filters were used to extract the five EEG frequency bands (i.e., delta, theta, alpha, beta, and gamma). Complexity measures were then obtained for each of the five EEG frequency bands and for each channel using each of the three methods of computing complexity measures (TsEn, HFD, and LZC).

For each method, we computed a panel of 114 biomarkers (i.e., 19 biomarkers for the whole EEG record and 19 biomarkers for each of the five EEG frequency bands). The performance measures for each biomarker were computed (including the sensitivity and specificity).

The paper is arranged as follows. In Section 2, the materials and methods used in the study are described. In Section 3, the results and discussions are presented and the conclusions are presented in Section 4.

\section{Materials and Methods}

2.1. Materials. This study was based on EEG dataset that was recorded from 52 volunteers. All the volunteers underwent a strict protocol based on normal hospital practices at Derriford Hospital, Plymouth, UK [11]. The EEG recordings include several states such as hyperventilation, awake, drowsy, and alert, with periods of eyes closed and open. For

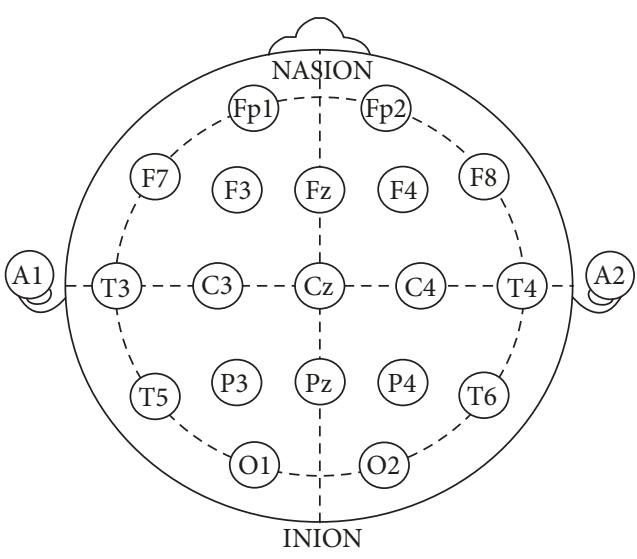

Figure 1: International 10-20 system.

storage reasons, the sampling rate was reduced from $256 \mathrm{~Hz}$ to $128 \mathrm{~Hz}$ by averaging two consecutive samples. The duration of each EEG signal is 4 minutes. Figure 1 shows the electrode locations using a 10-20 system. The letters F, C, P, O, and T refer to cerebral cortex lobes (F: frontal, C: central, P: parietal, O: occipital, and T: temporal) [82].

The EEG dataset consists of two subdatasets (A and B). Subdataset A includes 11 age matched subjects over 65 years old ( $3 \mathrm{AD}$ patients and 8 normal subjects). Subdataset A was recorded using the traditional 10-20 system in a Common Reference Montage by using the average of all channels as reference and the EEG signals were converted to Common Average and Bipolar Montages using software. Subdataset B includes 41 subjects that were not perfectly age matched ( 24 normal subjects, 10 males and 14 females, have mean age 69.4 \pm 11.5 years (from 40 to 84 years) and 17 were probable AD patients, 9 male and 8 female). The normal subjects have a mean age of $69.4 \pm 11.5$ years (40 to 84 years) and the probable AD subjects have mean age of $77.6 \pm 10.0$ years (from 50 to 93 years). Subdataset B was recorded using the modified Maudsley system. The conventional 10-20 system has a similar setting with the Maudsley electrode positioning system [83].

All patients were referred to the EEG department at Derriford Hospital from a specialist memory clinic. A battery of psychometric tests (including the MMSE [84], Rey Auditory Verbal Learning Test [85], Benton Visual Retention Test [86], and memory recall tests [87]) were performed on all patients at the memory clinic. The classification of subjects with dementia was based on the working diagnosis provided by the specialist memory clinic. All healthy volunteers and AD patients had their EEG confirmed by a consultant clinical neurophysiologist at the hospital as normal and probable mild $\mathrm{AD}$, respectively [11].

2.2. Methods. In our approach, the complete recordings of the EEG including artefacts were used without a priori selection of elements for analyses. This enabled us to have an idea about the robustness and usefulness of the method in practice. Data from a fixed interval (61s to $240 \mathrm{~s}$ ) was used to avoid electrical artefacts, which regularly occur at the 
beginning of a record, leaving a standard three-minute data to analyse.

The following steps outline the procedure that was used to derive the biomarkers for the three complexity methods (i.e., TsEn, HFD, and LZC)

(1) The EEG signal was filtered using infinite impulse response (IIR) Chebyshev-II bandpass filter into five frequency bands (i.e., delta $0-4 \mathrm{~Hz}$, theta $4-8 \mathrm{~Hz}$, alpha $8-12 \mathrm{~Hz}$, beta $12-30 \mathrm{~Hz}$, and gamma $30-45 \mathrm{~Hz}$ ). A low computational IIR filter was used to retain the computational efficiency of the derived complexitybased biomarkers [88].

(2) The biomarkers were then derived first from the whole EEG record and then for each of EEG frequency bands for each of the three EEG complexity methods.

(3) For each biomarker of the EEG complexity methods (i.e., TsEn, HFD, and LZC), $p$ values were computed between $\mathrm{AD}$ patients and normal subjects using Student's $t$-test.

(4) The performance of each complexity measure to detect $\mathrm{AD}$ is then assessed. For each complexity measure, a classification model, based on the support vector machine (SVM), was used to detect $A D$.

Tsallis Entropy (TsEn). TsEn [89] biomarker computation of an $N$-samples EEG data sequence $x(1), x(2), \ldots, x(N)$ is based on the generalised measure of entropy, due to Tsallis:

$$
\operatorname{TsEn}_{q}=\frac{\left(\sum_{i=1}^{k} P_{i}-P_{i}^{q}\right)}{(q-1)},
$$

where $\operatorname{TsEn}_{q}$ is the Tsallis entropy value, $k$ is the number of states that the amplitudes of the EEG are quantized into, $P_{i}$ is a probability associated with the $i$ th state, and $q$ is Tsallis parameter $(k=2200$ and $q=0.5)$.

Higuchi Fractal Dimension (HFD). To compute HFD biomarker $[75,77,90]$ of an $N$-sample EEG data sequence $x(1), x(2), \ldots, x(N)$, the data is first divided into a $k$-length subdata set as

$$
\begin{gathered}
x_{k}^{m}: x(m), x(m+k), x(m+2 k), \ldots, \\
x\left(m+\left[\frac{N-m}{k}\right] \cdot k\right),
\end{gathered}
$$

where [ ] is Gauss' notation, $k$ is constant, and $m=1,2, \ldots, k$. The length $L_{m}(k)$ for each subdata set is then computed as

$$
\begin{aligned}
& L_{m}(k) \\
& \quad=\left\{\left[\sum_{i=1}^{[(N-m) / k]}|x(m+i k)-x(m+(i-1) \cdot k)|\right]\right. \\
& \cdot((N-1) /([(N-m) / k] \cdot k))\} \cdot(k)^{-1} .
\end{aligned}
$$

The mean of $L_{m}(k)$ is then computed to find the HFD for the data as

$$
\mathrm{HFD}=\frac{1}{K} \sum_{M=1}^{K} L_{m}(k) .
$$

Lempel-Ziv Complexity (LZC). To compute the LZC [43, 49, $67,68,70]$ biomarker of an $N$-sample EEG data sequence $x(1), x(2), \ldots, x(N)$, the EEG signal is first converted into a binary string as

$$
x(i)= \begin{cases}0 & \text { if } \operatorname{EEG}(i)<M \\ 1 & \text { if } \operatorname{EEG}(i) \geq M\end{cases}
$$

where $x(i)$ is the equivalent binary value of $\operatorname{EEG}(i), i$ is the index of all values in the EEG signal, and $M$ is the median value of each EEG channel. The median value is used to manage the outliers.

The binary string is then scanned from left to right until the end to produce new substrings. A complexity counter $c(N)$ is the number of new substrings. The upper bound of $c(N)$ is used to normalise $c(N)$ to get an independent value from the sequence of length $N$. The upper bound of $c(N)$ is $N / \log _{2}(N) . c(N)$ is then normalised by $b(N)$ as

$$
C(N)=\frac{c(N)}{b(N)}
$$

where $C(N)$ is the normalised value of the LZC and $b(N)$ is the upper bound of the $c(N)$.

A panel of 114 biomarkers was computed (19 biomarkers for the whole EEG record and 19 biomarkers for each of EEG frequency band (i.e., delta, theta, alpha, beta, and gamma). To determine which features have a significant statistical association with $\mathrm{AD}$, we computed $p$ values between $\mathrm{AD}$ patients and normal subjects using Student's $t$-test. This allowed us to identify significant features that may be useful to discriminate between $\mathrm{AD}$ patients and normal subjects. The dataset was split into training and testing data (60\% for training and $40 \%$ for testing) with subjects selected at random. We selected 32 subjects for training and 20 subjects for testing at random from the datasets, a ratio of $60: 40$. The training data includes $12 \mathrm{AD}$ (two from dataset $\mathrm{A}$ and 10 from dataset $\mathrm{B}$ ) and 20 normal subjects (six from dataset $A$ and 14 from dataset B). The testing data includes $8 \mathrm{AD}$ (one from dataset $\mathrm{A}$ and seven from dataset B) and 12 normal subjects (two from dataset $\mathrm{A}$ and 10 from dataset $\mathrm{B})$. $p$ values were computed using the training EEG dataset. Machine learning techniques were used to develop models based on the biomarkers. As a classifier, we used support vector machine (SVM) to model biomarkers extracted using TsEn, HFD, and LZC methods. SVM classifier was used because it is widely used in machine learning and has found application in dementia diagnosis. It has shown better performance in biomedical data analysis and in automatic $\mathrm{AD}$ diagnosis compared to other conventional classifiers (e.g., Euclidean distance classifier) and good capability to learn from experimental data [91, 92], and it has a stable classification performance [93]. 
It has also been shown to outperform other machine learning techniques (e.g., Naive Bayes, Multilayer Perceptron, Bayes Network, egging, Logistic Regression, and Random Forest,) in diagnosis of MCI and dementia [94]. We used the testing EEG dataset to test the performance of the models. For each complexity method, six performance tables were created (whole EEG record, and table for each EEG frequency band).

The performance of the TsEn, HFD, and LZC biomarkers for AD diagnosis was assessed in terms of sensitivity (Sen), specificity (Spec), accuracy (ACC), F-measure, error rate, true positive rate (TPR), false positive rate (FPR), positive predictive value (PPV), and negative predictive value (NPV). Matthew's correlation coefficient (MCC) was computed to measure the quality of the binary classification (AD and normal) between the actual and predicted results $[95,96]$.

\section{Results and Discussions}

3.1. Result. We analysed the performance of the three different complexity measures in quantifying changes in EEG due to $\mathrm{AD}$. For this purpose, we examined the differences between the values of the complexity measures derived from EEG signals of $\mathrm{AD}$ subjects and those of normal subjects. Biomarkers that do not show significant differences between $\mathrm{AD}$ patients and normal subjects may not be suitable for quantifying changes in EEG due to $\mathrm{AD}$ as they may not be capable of being used to discriminate between $\mathrm{AD}$ patients and normal subjects.

We found that complexity measures derived from the EEG frequency bands for $\mathrm{AD}$ patients were significantly different to those of normal subjects compared to complexity measures derived from the whole EEG record. This suggests that they may be better suited to quantify changes in the EEG due to $\mathrm{AD}$ and potentially may provide better results in $\mathrm{AD}$ diagnosis.

Figure 2 shows the EEG biomarkers derived from whole EEG record (i.e., unfiltered) and those derived from the five EEG bands (delta, theta, alpha, beta, and gamma bands) using the TsEn method. The results show that TsEn values for $\mathrm{AD}$ patients are lower than those for normal subjects for whole EEG record. This is consistent with the findings in other studies [35, 58, 62, 64]. Figure 2 also shows that the differences between the TsEn values for $\mathrm{AD}$ patients and for normal subjects for the EEG bands (delta and theta bands in particular) are larger than those for whole EEG record. This is a desirable feature in a biomarker as it suggests that TsEn biomarkers derived from the EEG bands may provide better performance in detecting $\mathrm{AD}$ than those extracted from whole EEG record.

Figure 3 shows the EEG biomarkers derived from whole EEG record and those derived from the EEG bands (delta, theta, alpha, beta, and gamma bands in particular) using the HFD method. In this case, the results show that HFD values for $\mathrm{AD}$ patients are lower than those for normal subjects. This result is consistent with the finding in other studies $[46,80]$. As with the TsEn, the differences between HFD biomarkers for $\mathrm{AD}$ patients and normal subjects for the EEG frequency bands (i.e., delta, theta, and alpha bands) were

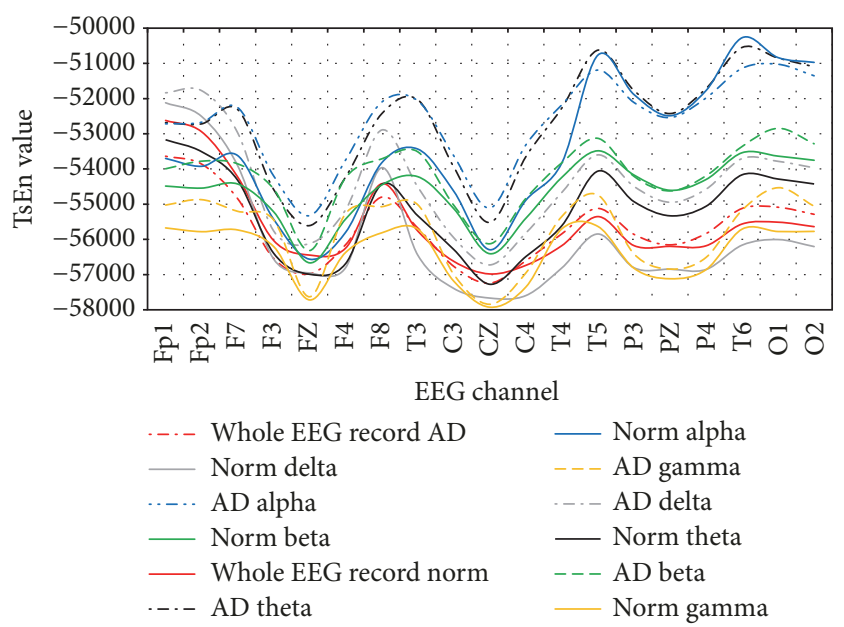

FIGURE 2: EEG biomarkers for TsEn.

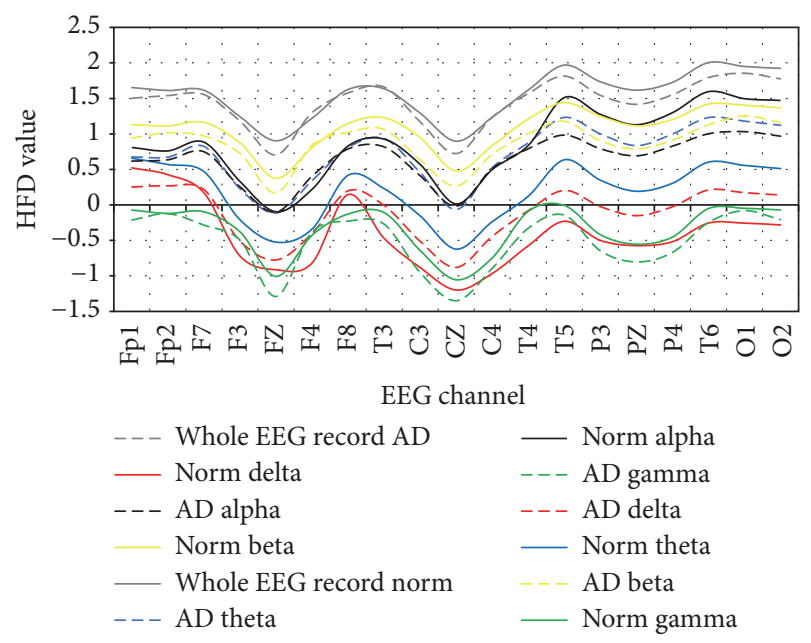

FIGURE 3: EEG biomarkers for HFD.

larger than those for the whole EEG record suggesting that the use of biomarkers derived from the frequency bands would be better at detecting $\mathrm{AD}$ than the use of whole EEG record.

Figure 4 shows similar results for the LZC method. In this case, the results show that LZC values for AD patients were lower than those for normal subjects and these are consistent with the finding in other studies [43, 97]. Again, the differences between the LZC biomarkers for AD patients and normal subjects for the five EEG frequency bands (the theta, beta, and gamma bands, in particular) were larger those for the whole EEG record, suggesting that the use of biomarkers derived from the frequency bands would be better at detecting $\mathrm{AD}$ than the use of whole EEG record.

We analysed the complexity measures using $p$ values to determine the statistical significance in detecting $\mathrm{AD}$

Figure 5 shows $p$ values of the differences in TsEn measures between $\mathrm{AD}$ patients and normal subjects for whole EEG record and those from the EEG frequency bands. The results show that TsEn biomarkers that were extracted from theta bands have the smallest $p$ values, while the TsEn 


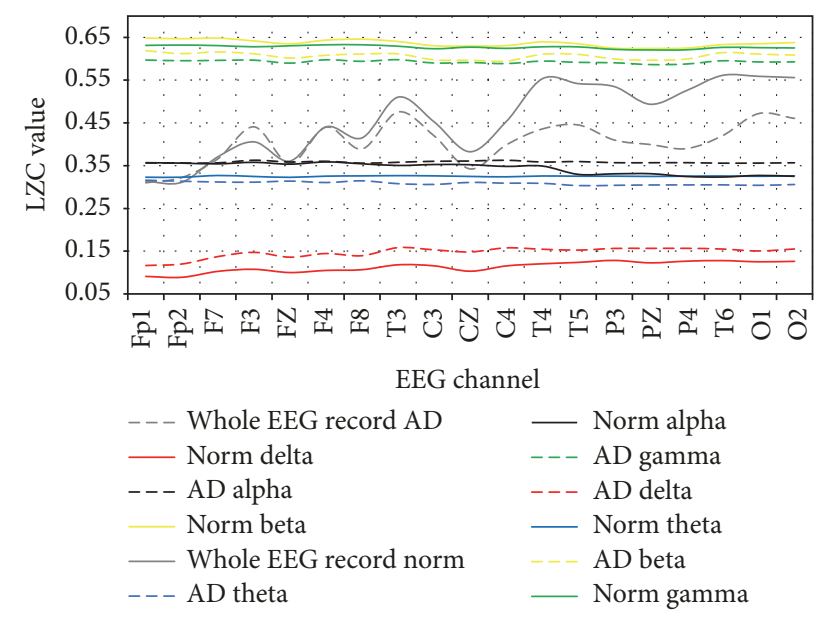

FIgURE 4: EEG biomarkers for LZC.

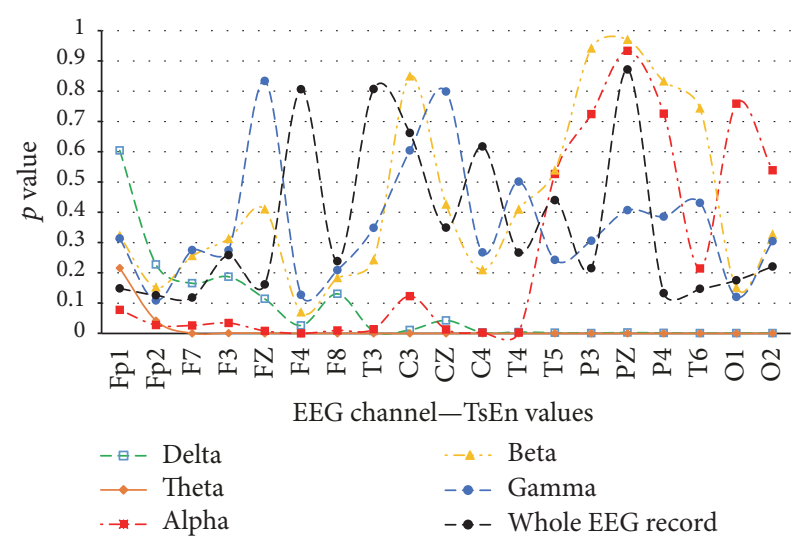

FIgURE 5: $p$ values for TsEn between AD patients and normal subjects of the training EEG dataset.

biomarkers derived from gamma band have the maximum $p$ value between $\mathrm{AD}$ patients and normal subjects. This suggests that biomarkers that are extracted from theta band may provide the best performance in $\mathrm{AD}$ diagnosis. Figure 5 also shows that biomarkers that were extracted from EEG frequency bands may have a more significant association with $\mathrm{AD}$ than the EEG biomarkers that are derived from whole EEG record based on $p$ value analysis. Therefore, the complexity measures derived from the EEG frequency band may provide better results in the classification between $A D$ patients and normal subjects.

Figures 6 and 7 depict the results of similar $p$ value analysis for HFD and LZC measures, respectively. The results show that, in both HFD and LZC methods, the complexity measures derived from the EEG frequency bands, theta band have significantly smaller $p$ values compared to those of measures derived from the whole EEG record. In both methods, complexity measures derived from the theta band gave the smallest $p$ value. This implies that biomarkers derived from the frequency bands, the theta band in particular, may provide the best possible performance in $\mathrm{AD}$ diagnosis using the HFD and LZC methods.

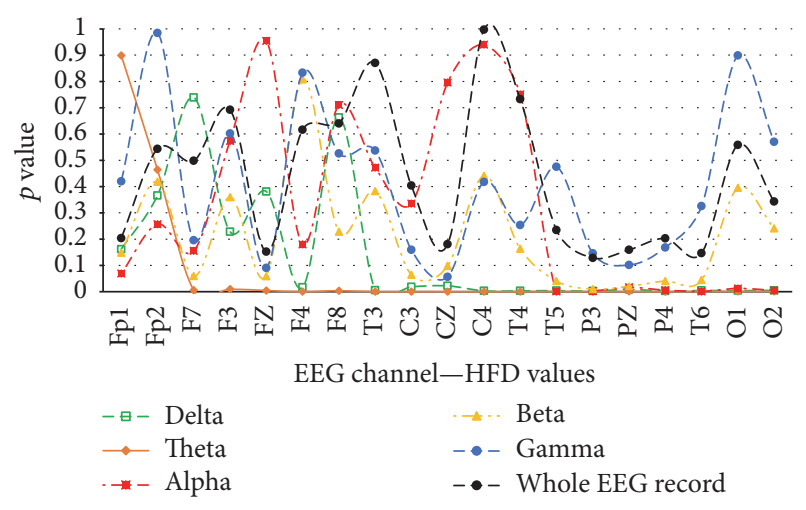

FIGURE 6: $p$ values for HFD between AD patients and normal subjects of the training EEG dataset.

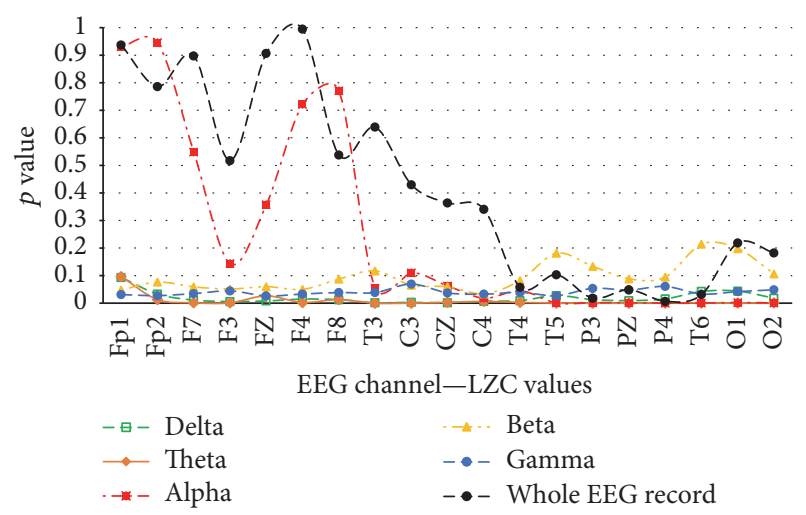

FIgURE 7: $p$ values for LZC between AD patients and normal subjects of the training EEG dataset.

Looking across all the results (Figures 5, 6, and 7), the theta band has a minimum $p$ value between $\mathrm{AD}$ patients and normal subjects for all three complexity methods (i.e., TsEn, HFD, and LZC). Thus, EEG biomarkers derived from EEG frequency bands are better than the biomarkers that were extracted from whole EEG record. The biomarkers derived from theta band may provide the best performance in $\mathrm{AD}$ diagnosis across all three methods.

3.2. The Performance of the EEG Complexity-Based Measures. Table 1 shows the performance of the SVM-based classification model using TsEn biomarkers for whole EEG record for the 19 EEG channels. In this case, the best sensitivity and specificity were $46.67 \%$ and $80 \%$, respectively, for Fp2 and F7 EEG channels.

Similar performance indices were computed for each of the five EEG bands using the TsEn. As an example, Table 2 shows the performance indices for TsEn biomarkers for the delta band for the 19 EEG channels. The best sensitivity and specificity were $85.71 \%$ and $84.62 \%$, respectively, for T4, O1, and O2 EEG channels.

Similar performance indices were computed for each of the five EEG bands using HFD and LZC methods. Table 3 
TABLE 1: TsEn performance for whole EEG record.

\begin{tabular}{lcccccccccc}
\hline EEG channel & Sen.\% & Spec. $\%$ & Acc. $\%$ & F-measure\% & Error rate & MCC & FPR\% & FNR\% & PPV\% & NPV\% \\
\hline Fp1 & 43.75 & 75.00 & 50.00 & 58.33 & 0.50 & 0.153 & 25.00 & 56.25 & 87.50 & 25.00 \\
Fp2 & 46.67 & 80.00 & 55.00 & 60.87 & 0.45 & 0.236 & 20.00 & 53.33 & 87.50 & 33.33 \\
F7 & 46.67 & 80.00 & 55.00 & 60.87 & 0.45 & 0.236 & 20.00 & 53.33 & 87.50 & 33.33 \\
F3 & 43.75 & 75.00 & 50.00 & 58.33 & 0.50 & 0.153 & 25.00 & 56.25 & 87.50 & 25.00 \\
FZ & 44.44 & 100.00 & 50.00 & 61.54 & 0.50 & 0.272 & 0.00 & 55.56 & 100.00 & 16.67 \\
F4 & 44.44 & 100.00 & 50.00 & 61.54 & 0.50 & 0.272 & 0.00 & 55.56 & 100.00 & 16.67 \\
F8 & 44.44 & 100.00 & 50.00 & 61.54 & 0.50 & 0.272 & 0.00 & 55.56 & 100.00 & 16.67 \\
T3 & 37.50 & 50.00 & 40.00 & 50.00 & 0.60 & -0.102 & 50.00 & 62.50 & 75.00 & 16.67 \\
C3 & 35.71 & 50.00 & 40.00 & 45.45 & 0.60 & -0.134 & 50.00 & 64.29 & 62.50 & 25.00 \\
CZ & 42.11 & 100.00 & 45.00 & 59.26 & 0.55 & 0.187 & 0.00 & 57.89 & 100.00 & 8.33 \\
C4 & 44.44 & 100.00 & 50.00 & 61.54 & 0.50 & 0.272 & 0.00 & 55.56 & 100.00 & 16.67 \\
T4 & 35.29 & 33.33 & 35.00 & 48.00 & 0.65 & -0.229 & 66.67 & 64.71 & 75.00 & 8.33 \\
T5 & 33.33 & 50.00 & 40.00 & 40.00 & 0.60 & -0.167 & 50.00 & 66.67 & 50.00 & 33.33 \\
P3 & 28.57 & 33.33 & 30.00 & 36.36 & 0.70 & -0.356 & 66.67 & 71.43 & 50.00 & 16.67 \\
PZ & 37.50 & 50.00 & 40.00 & 50.00 & 0.60 & -0.102 & 50.00 & 62.50 & 75.00 & 16.67 \\
P4 & 35.71 & 50.00 & 40.00 & 45.45 & 0.60 & -0.134 & 50.00 & 64.29 & 62.50 & 25.00 \\
T6 & 26.67 & 20.00 & 25.00 & 34.78 & 0.75 & -0.471 & 80.00 & 73.33 & 50.00 & 8.33 \\
O1 & 27.27 & 44.44 & 35.00 & 31.58 & 0.65 & -0.287 & 55.56 & 72.73 & 37.50 & 33.33 \\
O2 & 30.00 & 50.00 & 40.00 & 33.33 & 0.60 & -0.204 & 50.00 & 70.00 & 37.50 & 41.67 \\
\hline
\end{tabular}

TABLE 2: TsEn performance for delta band of the EEG signal.

\begin{tabular}{lcccccccccc}
\hline EEG channel & Sen.\% & Spec.\% & Acc.\% & F-measure\% & Error rate & MCC & FPR\% & FNR\% & PPV\% & NPV\% \\
\hline Fp1 & 50.00 & 66.67 & 60.00 & 50.00 & 0.40 & 0.167 & 33.33 & 50.00 & 50.00 & 66.67 \\
Fp2 & 50.00 & 62.50 & 60.00 & 33.33 & 0.40 & 0.102 & 37.50 & 50.00 & 25.00 & 83.33 \\
F7 & 55.56 & 72.73 & 65.00 & 58.82 & 0.35 & 0.287 & 27.27 & 44.44 & 62.50 & 66.67 \\
F3 & 80.00 & 73.33 & 75.00 & 61.54 & 0.25 & 0.471 & 26.67 & 20.00 & 50.00 & 91.67 \\
FZ & 50.00 & 62.50 & 60.00 & 33.33 & 0.40 & 0.102 & 37.50 & 50.00 & 25.00 & 83.33 \\
F4 & 50.00 & 61.11 & 60.00 & 20.00 & 0.40 & 0.068 & 38.89 & 50.00 & 12.50 & 91.67 \\
F8 & 57.14 & 69.23 & 65.00 & 53.33 & 0.35 & 0.257 & 30.77 & 42.86 & 50.00 & 75.00 \\
T3 & 71.43 & 76.92 & 75.00 & 66.67 & 0.25 & 0.471 & 23.08 & 28.57 & 62.50 & 83.33 \\
C3 & 60.00 & 66.67 & 65.00 & 46.15 & 0.35 & 0.236 & 33.33 & 40.00 & 37.50 & 83.33 \\
CZ & 100.00 & 63.16 & 65.00 & 22.22 & 0.35 & 0.281 & 36.84 & 0.00 & 12.50 & 100.00 \\
C4 & 71.43 & 76.92 & 75.00 & 66.67 & 0.25 & 0.471 & 23.08 & 28.57 & 62.50 & 83.33 \\
T4 & 85.71 & 84.62 & 85.00 & 80.00 & 0.15 & 0.685 & 15.38 & 14.29 & 75.00 & 91.67 \\
T5 & 80.00 & 73.33 & 75.00 & 61.54 & 0.25 & 0.471 & 26.67 & 20.00 & 50.00 & 91.67 \\
P3 & 75.00 & 83.33 & 80.00 & 75.00 & 0.20 & 0.583 & 16.67 & 25.00 & 75.00 & 83.33 \\
PZ & 100.00 & 75.00 & 80.00 & 66.67 & 0.20 & 0.612 & 25.00 & 0.00 & 50.00 & 100.00 \\
P4 & 83.33 & 78.57 & 80.00 & 71.43 & 0.20 & 0.579 & 21.43 & 16.67 & 62.50 & 91.67 \\
T6 & 83.33 & 78.57 & 80.00 & 71.43 & 0.20 & 0.579 & 21.43 & 16.67 & 62.50 & 91.67 \\
O1 & 85.71 & 84.62 & 85.00 & 80.00 & 0.15 & 0.685 & 15.38 & 14.29 & 75.00 & 91.67 \\
O2 & 85.71 & 84.62 & 85.00 & 80.00 & 0.15 & 0.685 & 15.38 & 14.29 & 75.00 & 91.67 \\
\hline
\end{tabular}

summarises the best performance indices for the three complexity measures.

Figures 8, 9, and 10 summarise the performance indices of the TsEn, HFD, and ZLC methods.

The results show that TsEn, HFD, and ZLC EEG biomarkers derived from the EEG frequency bands provide better performance than EEG biomarkers derived from the whole EEG record.
3.3. Discussions. The results of this study show that EEG complexity-based measures provide a potentially useful way to detect $\mathrm{AD}$. The most characteristic feature caused by $\mathrm{AD}$ is the reduction in EEG complexity [33-35, 37, 38, 40, 41] compared to normal subjects. This is consistent with other studies $[35,38,43,46,56,58,62,64,80,97,98]$ and shows that EEG complexity measures are potentially a good biomarker for detecting $\mathrm{AD}$. 


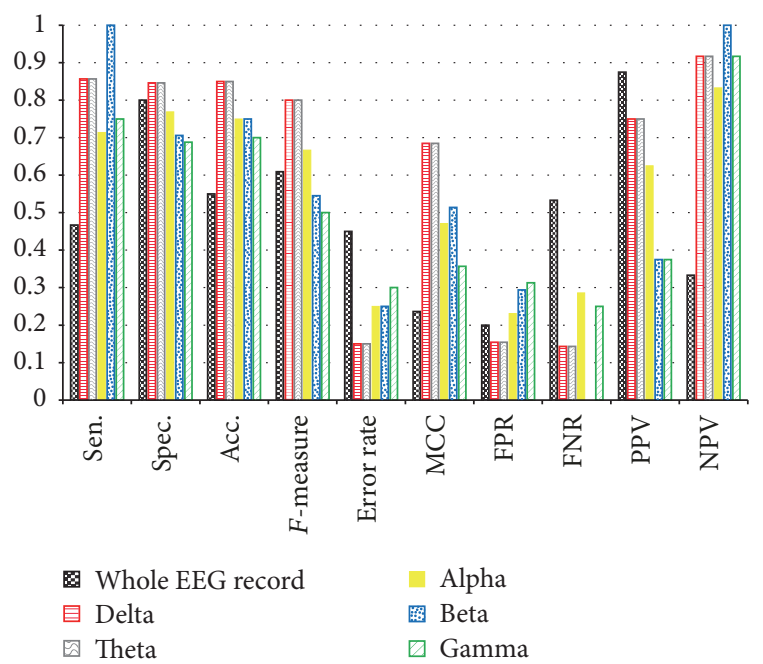

FIGURE 8: TsEn performance.

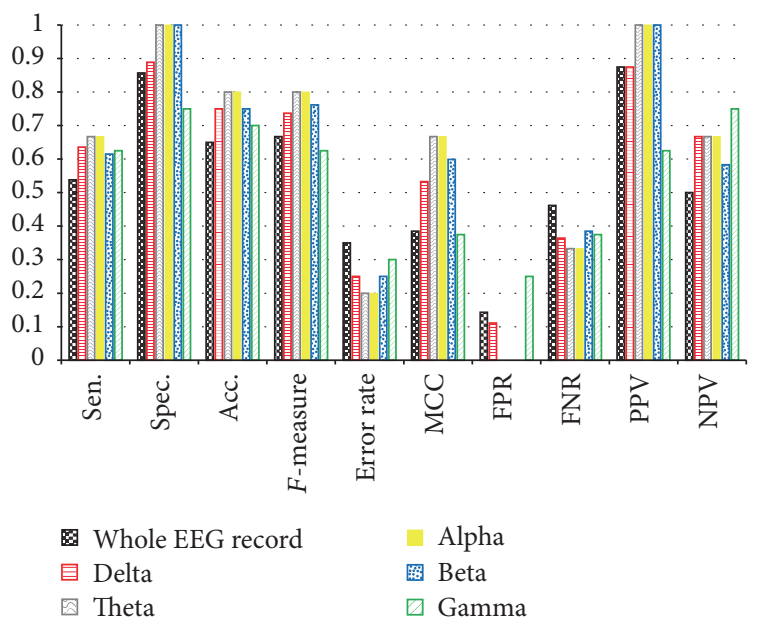

FIGURE 9: HFD performance.

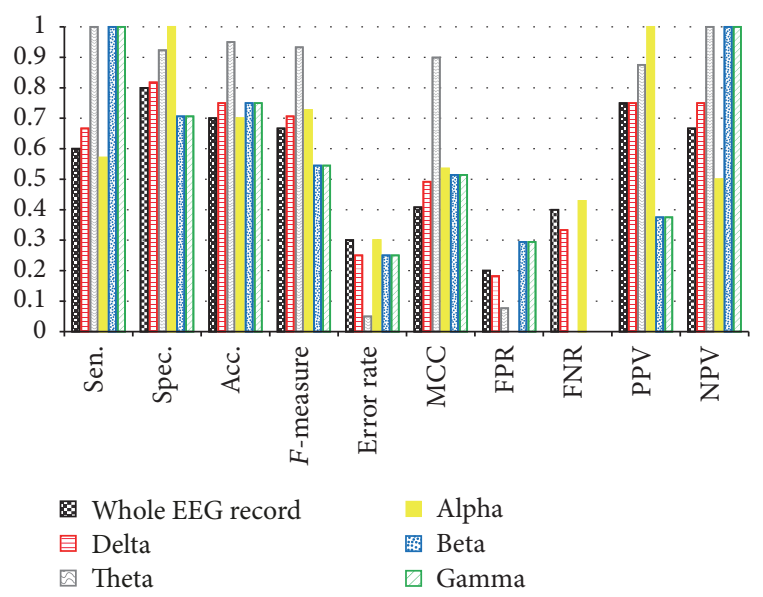

FIGURE 10: LZC performance.
TABLE 3: Summary of the best performance indices for the three complexity measures.

\begin{tabular}{lccccc}
\hline Method & \multicolumn{2}{c}{ TsEn } & \multicolumn{2}{c}{ HFD } & LZC \\
\hline Feature & Delta & Theta & Theta & Alpha & Theta \\
EEG channel & T4, O1, O2 & F4 & C4 & T5, P3 & C3 \\
Sen.\% & 85.71 & 85.71 & 66.67 & 66.67 & 100 \\
Spec.\% & 84.62 & 84.62 & 100 & 100 & 92.31 \\
Acc.\% & 85 & 85 & 80 & 80 & 95 \\
F-measure\% & 80 & 80 & 80 & 80 & 93.33 \\
Error rate & 0.15 & 0.15 & 0.2 & 0.2 & 0.05 \\
MCC & 0.685 & 0.685 & 0.667 & 0.667 & 0.9 \\
FPR\% & 15.38 & 15.38 & 0 & 0 & 7.69 \\
FNR\% & 14.29 & 14.29 & 33.33 & 33.33 & 0 \\
PPV\% & 75 & 75 & 100 & 100 & 87.5 \\
NPV\% & 91.67 & 91.67 & 66.67 & 66.67 & 100 \\
\hline
\end{tabular}

Unlike previous studies, we found that the complexity measures derived from the EEG frequency bands (i.e., delta, theta, alpha, beta, and gamma) provide significantly better performance in detecting $\mathrm{AD}$ than the complexity measures derived from whole EEG records. This comes from the greater differences between the complexity measures for $\mathrm{AD}$ patients and normal subjects when they are derived from the frequency bands compared to when they are derived from whole record which is a desirable property of a good biomarker.

In particular, we found that for the TsEn and HFD complexity measures derived from the delta and theta bands gave the best performance. For the delta band, three EEG channels (T4, O1, and O2) gave the best performance. For the theta band, $\mathrm{F} 4$ gave the best performance.

Similar results were obtained for the LZC complexity measures, except that the best EEG channel was C3 for the theta band. This is consistent with the findings of other studies which suggested that $\mathrm{AD}$ starts from the back of the brain and then spreads gradually to other parts of the brain [5, 46, 99-101]. This implies that it may be possible to use only a small number of EEG channels to detect AD.

The findings of this study have a number of implications for research to develop new and robust techniques for the analysis of EEG to increase the contributions EEG makes to the diagnosis of $\mathrm{AD}$.

The results suggest that the three EEG complexity measures, derived from the EEG frequency bands, can detect $\mathrm{AD}$ reliably (with sensitivity and specificity of $>90 \%$ ). Thus, EEG complexity measures could provide a basis for developing an accurate, low-cost, and easy to use tool to detect AD. Although the results of the studies are consistent with previous studies, unlike previous studies, in this study, the complexity measures are derived from EEG frequency bands (i.e., delta, theta, alpha, beta, and gamma). The results suggest that deriving the complexity measures from the EEG frequency bands is an important step for achieving robust biomarkers.

We found that $\mathrm{AD}$ patients have significantly lower complexity measures for specific EEG frequency bands and for specific EEG channels than normal subjects. This is 
consistent with findings in previous studies [33-35, 37, 38, $40,41]$. Thus, it may be possible to identify specific EEG channels and specific frequency bands that may provide the best biomarkers to detect $\mathrm{AD}$. In situations where the number of available channels is limited (e.g., when portable EEG systems are used outside specialist centres), this may be exploited to achieve a good performance.

It may be possible to enhance the performance of the complexity-based approach further, by combining the three complexity measures into a composite model. Given that the three complexity measures are analysing different aspects of the signal (e.g., entropy and fractal measures), integrating them may lead to improved performance.

Our study has a number of limitations. At present, our methods have been applied only to the detection of $\mathrm{AD}$, the most common form of dementia. A more detailed study is necessary to evaluate the methods using a much larger and diverse EEG datasets. This includes using the methods to differentiate between normal, MCI, and AD subjects $[57,63,73]$.

This study shows that the abnormalities caused by $\mathrm{AD}$ can be detected by the complexity measures. However, similar changes may be caused by other neurodegenerative diseases, such as other types of dementia. To enhance the diagnostic usefulness of the methods, it may be necessary to develop them further to differentiate between dementias.

\section{Conclusions}

$\mathrm{AD}$ causes changes in the EEG due to loss of memory and cognitive decline and these changes are thought to be associated with functional disconnections among cortical areas resulting from the death of brain cells. Therefore, EEG analysis may provide valuable information about brain dynamics in $\mathrm{AD}$. $\mathrm{AD}$ causes a reduction in neuronal activity of the brain and this may be reflected in EEG signals. Nonlinear methods based on EEG complexity approaches have shown promising results in detected changes in the EEG thought to be due to AD. Therefore, EEG complexity can potentially be a good biomarker for $\mathrm{AD}$ diagnosis. We investigated three complexity measures, TsEn, HFD, and LZC methods, derived from EEG frequency bands. We found that AD patients have significantly lower TsEn, HFD, and LZC values in specific EEG frequency bands and specific EEG channels compared to normal subjects. This may provide an effective way to discriminate between $\mathrm{AD}$ patients and normal subjects. Future work will evaluate the methods using larger and more diverse EEG datasets, including different types of dementia.

\section{Conflicts of Interest}

The authors declare that there are no conflicts of interest regarding the publication of this article

\section{Acknowledgments}

The first author would like to thank the Ministry of Higher Education and Scientific Research (MoHESR), Iraq, for their financial support. Financial support by the EPSRC is also gratefully acknowledged.

\section{References}

[1] T. Harmony, T. Fernández, J. Gersenowies et al., "Specific EEG frequencies signal general common cognitive processes as well as specific task processes in man," International Journal of Psychophysiology, vol. 53, no. 3, pp. 207-216, 2004.

[2] C. Humpel, "Identifying and validating biomarkers for Alzheimer's disease," Trends in Biotechnology, vol. 29, no. 1, pp. 26-32, 2011.

[3] W. H. Organization, WHO Mental Health Gap Action Programme (mhGAP) 2008, 2014.

[4] ADNI, http://www.adni-info.org/.

[5] A. H. Al-Nuaimi, E. Jammeh, L. Sun, and E. Ifeachor, "Changes in the EEG amplitude as a biomarker for early detection of Alzheimer's disease," in Proceedings of the 38th Annual International Conference of the IEEE Engineering in Medicine and Biology Society, EMBC 2016, pp. 993-996, USA, August 2016.

[6] M. Prince, A. Wimo, M. Guerchet, G.-C. Ali, Y. Wu, and M. Prina, World Alzheimer Report, 2015.

[7] M. Prince, A. Comas-Herrera, M. Knapp, M. Guerchet, and M. Karagiannidou, "World Alzheimer Report 2016 Improving healthcare for people living with dementia," Coverage, Quality and costs now and in the future, pp. 1-140, 2016.

[8] E. Jammeh, C. Carroll, S. Pearson et al., "Using NHS primary care data to identify undiagnosed dementia," Journal of Neurology, Neurosurgery \& Psychiatry, vol. 86, no. 11, pp. e4.134-e4, 2015.

[9] B. Michalowsky, T. Eichler, J. R. Thyrian et al., "Healthcare resource utilization and cost in dementia: Are there differences between patients screened positive for dementia with and those without a formal diagnosis of dementia in primary care in Germany?" International Psychogeriatrics, vol. 28, no. 3, pp. 359-369, 2016.

[10] M. Prince, R. Bryce, and C. Ferri, The benefits of early diagnosis and intervention World Alzheimer Report 2011, Elsevier, 2011.

[11] G. Henderson, E. Ifeachor, N. Hudson et al., "Development and assessment of methods for detecting dementia using the human electroencephalogram," IEEE Transactions on Biomedical Engineering, vol. 53, no. 8, pp. 1557-1568, 2006.

[12] A. L. Sutton, Ed., Alzheimer Disease Sourcebook, Omnigraphics: Peter E. Ruffner, Detroit, USA, 5th edition, 2011.

[13] D. Galimberti and E. Scarpani, Biomarkers for Early Diagnosis of Alzheimer's Disease, Nova Biomedical Books, 2008.

[14] A. Association, 2017 Alzheimer's disease facts and figures, Alzheimer's Dement, 2017.

[15] K. A. Jellinger, B. Janetzky, J. Attems, and E. Kienzl, "Biomarkers for early diagnosis of Alzheimer disease: 'ALZheimer ASsociated gene' - A new blood biomarker?" Journal of Cellular and Molecular Medicine, vol. 12, no. 4, pp. 1094-1117, 2008.

[16] K. Ritchie and S. Lovestone, "The dementias," The Lancet, vol. 360, no. 9347, pp. 1759-1766, 2002.

[17] R. A. Sperling, P. S. Aisen, L. A. Beckett et al., ““Toward defining the preclinical stages of Alzheimer's disease: Recommendations from the National Institute on Aging-Alzheimer's Association workgroups on diagnostic guidelines for Alzheimer's disease," Alzheimer's Dement, vol. 7, no. 3, pp. 333-334, 2011. 
[18] A. Association, "2016 Alzheimer's disease facts and figures," Alzheimer's Dement, vol. 12, no. 4, pp. 459-509, 2016.

[19] M. D. Greicius, B. Krasnow, A. L. Reiss, and V. Menon, "Functional connectivity in the resting brain: a network analysis of the default mode hypothesis," Proceedings of the National Acadamy of Sciences of the United States of America, vol. 100, no. 1, pp. 253-258, 2003.

[20] A. Gevins, M. E. Smith, L. McEvoy, and D. Yu, "High-resolution EEG mapping of cortical activation related to working memory: effects of task difficulty, type of processing, and practice," Cerebral Cortex, vol. 7, no. 4, pp. 374-385, 1997.

[21] K. Sasaki, A. Nambu, T. Tsujimoto, R. Matsuzaki, S. Kyuhou, and H. Gemba, "Studies on integrative functions of the human frontal association cortex with MEG," Cognitive Brain Research, vol. 5, no. 1-2, pp. 165-174, 1996.

[22] T. Abeel, T. Helleputte, Y. Van de Peer, P. Dupont, and Y. Saeys, "Robust biomarker identification for cancer diagnosis with ensemble feature selection methods," Bioinformatics, vol. 26, no. 3, pp. 392-398, 2009.

[23] A. Boucheham and M. Batouche, "Robust biomarker discovery for cancer diagnosis based on meta-ensemble feature selection," in Proceedings of the 2014 Science and Information Conference, SAI 2014, pp. 452-460, UK, August 2014.

[24] A. H. Al-nuaimi, E. Jammeh, L. Sun, and E. Ifeachor, "Changes in the Electroencephalogram as a Biomarker of Alzheimer's Disease," in Proceedings of the Biosense Dementia 2017 - international workshop on biosensors for dementia from 13 - 14 June 2017, Plymouth University, Plymouth, UK, 2017.

[25] A. Cedazo-Minguez and B. Winblad, "Biomarkers for Alzheimer's disease and other forms of dementia: clinical needs, limitations and future aspects," Experimental Gerontology, vol. 45, no. 1, pp. 5-14, 2010.

[26] D. Ferreira, V. Jelic, L. Cavallin et al., "Electroencephalography Is a Good Complement to Currently Established Dementia Biomarkers," Dementia and Geriatric Cognitive Disorders, vol. 42, no. 1-2, pp. 80-92, 2016.

[27] K. Blennow and H. Hampel, "CSF markers for incipient Alzheimer's disease," The Lancet Neurology, vol. 2, no. 10, pp. 605-613, 2003.

[28] S. E. O’Bryant, M. M. Mielke, R. A. Rissman et al., "Bloodbased biomarkers in Alzheimer disease: Current state of the science and a novel collaborative paradigm for advancing from discovery to clinic," Alzheimer's \& Dementia, vol. 13, no. 1, pp. 45-58, 2017.

[29] D. Ferreira, L. Perestelo-Pérez, E. Westman, L.-O. Wahlund, A. Sarrisa, and P. Serrano-Aguilar, "Meta-review of CSF core biomarkers in Alzheimer's disease: The state-of-the-art after the new revised diagnostic criteria," Frontiers in Aging Neuroscience, vol. 6, Article ID Article 47, 2014.

[30] M. Weiner and Z. Khachaturian, The use of MRI and PET for clinical diagnosis of dementia and investigation of cognitive impairment: a consensus report, Alzheimer's Assoc., Chicago, USA, 2005.

[31] B. Dubois, H. Hampel, H. H. Feldman et al., "Preclinical Alzheimer's disease: definition, natural history, and diagnostic criteria," Alzheimer's \& Dementia, vol. 12, no. 3, pp. 292-323, 2016.

[32] H. Jiang, M. P. White, M. D. Greicius, L. C. Waelde, and D. Spiegel, "Brain activity and functional connectivity associated with hypnosis," Cerebral Cortex, vol. 27, no. 8, pp. 4083-4093, 2017.
[33] M. Signorino, E. Pucci, N. Belardinelli, G. Nolfe, and F. Angeleri, "EEG spectral analysis in vascular and Alzheimer dementia," Electroencephalography and Clinical Neurophysiology, vol. 94, no. 5, pp. 313-325, 1995.

[34] C. Besthorn, R. Zerfass, C. Geiger-Kabisch et al., "Discrimination of Alzheimers disease and normal aging by EEG data," Electroencephalography and Clinical Neurophysiology, vol. 103, no. 2, pp. 241-248, 1997.

[35] A. H. Al-Nuaimi, E. Jammeh, L. Sun, and E. Ifeachor, “Tsallis entropy as a biomarker for detection of Alzheimer's disease," in Proceedings of the 37th Annual International Conference of the IEEE Engineering in Medicine and Biology Society, EMBC 2015, pp. 4166-4169, Italy, August 2015.

[36] A. Horvath, A. Szucs, G. Csukly, A. Sakovics, G. Stefanics, and A. Kamondi, EEG and ERP biomarkers of Alzheimer's disease: $a$ critical review, EEG and ERP biomarkers of Alzheimer's disease, a critical review.

[37] D. Moretti, C. Fracassi, M. Pievani et al., "Increase of theta/gamma ratio is associated with memory impairment," Clinical Neurophysiology, vol. 120, no. 2, pp. 295-303, 2009.

[38] J. Jeong, "EEG dynamics in patients with Alzheimer's disease," Clinical Neurophysiology, vol. 115, no. 7, pp. 1490-1505, 2004.

[39] Y. A. L. Pijnenburg, Y. Vd Made, A. M. van Cappellen van Walsum, D. L. Knol, P. Scheltens, and C. J. Stam, "EEG synchronization likelihood in mild cognitive impairment and Alzheimer's disease during a working memory task," Clinical Neurophysiology, vol. 115, no. 6, pp. 1332-1339, 2004.

[40] M. Ishikawa, K. Doya, H. Miyamoto, and T. Yamakawa, "Dynamical nonstationarity analysis of resting EEGs in Alzheimer's disease," in Proceedings of the International Conference on Neural Information Processing, pp. 921-929, 2007.

[41] J. Dauwels, F.-B. Vialatte, and A. Cichocki, "On the early diagnosis of Alzheimer's disease from EEG signals: a minireview," in Advances in Cognitive Neurodynamics (II), pp. 709716, Springer, 2011.

[42] C. Babiloni, G. Binetti, E. Cassetta et al., "Sources of cortical rhythms change as a function of cognitive impairment in pathological aging: a multicenter study," Clinical Neurophysiology, vol. 117 , no. 2, pp. 252-268, 2006.

[43] D. Abásolo, R. Hornero, C. Gómez, M. García, and M. López, "Analysis of EEG background activity in Alzheimer's disease patients with Lempel-Ziv complexity and central tendency measure," Medical Engineering \& Physics, vol. 28, no. 4, pp. 315322, 2006.

[44] C. Babiloni, C. Del Percio, F. Vecchio et al., "Alpha, beta and gamma electrocorticographic rhythms in somatosensory, motor, premotor and prefrontal cortical areas differ in movement execution and observation in humans," Clinical Neurophysiology, vol. 127, no. 1, pp. 641-654, 2016.

[45] G. G. Yener, D. D. Emek-Savaş, R. Lizio et al., "Frontal delta event-related oscillations relate to frontal volume in mild cognitive impairment and healthy controls," International Journal of Psychophysiology, vol. 103, pp. 110-117, 2016.

[46] A. H. Al-Nuaimi, E. Jammeh, L. Sun, and E. Ifeachor, "Higuchi fractal dimension of the electroencephalogram as a biomarker for early detection of Alzheimer's disease," in Proceedings of the 2017 39th Annual International Conference of the IEEE Engineering in Medicine and Biology Society (EMBC), pp. 23202324, Jeju Island, South Korea, July 2017.

[47] D. Abásolo, R. Hornero, P. Espino, J. Poza, C. I. Sánchez, and R. De La Rosa, "Analysis of regularity in the EEG background 
activity of Alzheimer's disease patients with Approximate Entropy," Clinical Neurophysiology, vol. 116, no. 8, pp. 1826-1834, 2005.

[48] H. Kantz and T. Schreiber, Nonlinear Time Series Analysis, Cambridge University Press, 2nd edition, 2004.

[49] A. Lempel and J. Ziv, "On the Complexity of Finite Sequences," IEEE Transactions on Information Theory, vol. 22, no. 1, pp. 7581, 1976.

[50] G. Tononi, G. M. Edelman, and O. Sporns, "Complexity and coherency: integrating information in the brain," Trends in Cognitive Sciences, vol. 2, no. 12, pp. 474-484, 1998.

[51] R. Wackerbauer, A. Witt, H. Atmanspacher, J. Kurths, and H. Scheingraber, "A comparative classification of complexity measures," Chaos, Solitons \& Fractals, vol. 4, no. 1, pp. 133-173, 1994.

[52] C. Besthorn, H. Sattel, C. Geiger-Kabisch, R. Zerfass, and H. Förstl, "Parameters of EEG dimensional complexity in Alzheimer's disease," Electroencephalography and Clinical Neurophysiology, vol. 95, no. 2, pp. 84-89, 1995.

[53] A. P. Burgess, J. Rehman, and J. D. Williams, "Changes in neural complexity during the perception of 3D images using random dot stereograms," International Journal of Psychophysiology, vol. 48, no. 1, pp. 35-42, 2003.

[54] F. Gu, X. Meng, E. Shen, and Z. Cai, "Can we measure consciousness with EEG complexities?" International Journal of Bifurcation and Chaos, vol. 13, no. 3, pp. 733-742, 2003.

[55] H. Adeli and S. Ghosh-Dastidar, Automated EEG-based diagnosis of neurological disorders: Inventing the future of neurology, CRC Press, 2010.

[56] J. C. McBride, X. Zhao, N. B. Munro et al., "Spectral and complexity analysis of scalp EEG characteristics for mild cognitive impairment and early Alzheimer's disease," Computer Methods and Programs in Biomedicine, vol. 114, no. 2, pp. 153-163, 2014.

[57] J. Dauwels, K. Srinivasan, M. Ramasubba Reddy et al., "Slowing and loss of complexity in Alzheimer's EEG: Two sides of the same coin?" International Journal of Alzheimer's Disease, Article ID 539621, 2011.

[58] P. Zhao, P. Van-Eetvelt, C. Goh, N. Hudson, S. Wimalaratna, and E. Ifeachor, "Characterization of EEGs in Alzheimer's disease using information theoretic methods," in Proceedings of the 29th Annual International Conference of the IEEEon Engineering in Medicine and Biology Society (EMBC '07), pp. 5127-5131, Lyon, France, August 2007.

[59] B. Hamadicharef, C. Guan, N. Hudson, E. C. Ifeachor, and S. Wimalaratna, "Performance evaluation and fusion of methods for early detection of Alzheimer Disease," in Proceedings of the 1st International Conference on BioMedical Engineering and Informatics (BMEI '08), pp. 347-351, Sanya, China, May 2008.

[60] J. Escudero, D. Abásolo, R. Hornero, P. Espino, and M. López, "Analysis of electroencephalograms in Alzheimer's disease patients with multiscale entropy," Physiological Measurement, vol. 27, no. 11, article 004, p. 1091, 2006.

[61] M. Zanin, L. Zunino, O. A. Rosso, and D. Papo, "Permutation entropy and its main biomedical and econophysics applications: a review," Entropy, vol. 14, no. 8, pp. 1553-1577, 2012.

[62] T. J. De Bock, S. Das, M. Mohsin et al., "Early detection of Alzheimer's disease using nonlinear analysis of EEG via Tsallis entropy," in Proceedings of the 2010 Biomedical Sciences and Engineering Conference (BSEC), pp. 1-4, Oak Ridge, TN, USA, 2010.
[63] J. Dauwels, F. Vialatte, and A. Cichocki, "Diagnosis of Alzheimer's disease from EEG signals: where are we standing?" Current Alzheimer Research, vol. 7, no. 6, pp. 487-505, 2010.

[64] C. Coronel, H. Garn, M. Waser et al., "Quantitative EEG markers of entropy and auto mutual information in relation to MMSE scores of probable Alzheimer's disease patients," Entropy, vol. 19, no. 3, article no. 130, 2017.

[65] R. Sneddon, W. R. Shankle, J. Hara, A. Rodriquez, D. Hoffman, and U. Saha, "EEG Detection of Early Alzheimer's Disease Using Psychophysical Tasks," Clinical EEG and Neuroscience, vol. 36, no. 3, pp. 141-150, 2005.

[66] H. Garn, M. Waser, M. Deistler et al., "Quantitative EEG markers relate to Alzheimer's disease severity in the Prospective Dementia Registry Austria (PRODEM)," Clinical Neurophysiology, vol. 126, no. 3, pp. 505-513, 2015.

[67] D. Abásolo, S. Simons, R. Morgado da Silva, G. Tononi, and V. V. Vyazovskiy, "Lempel-Ziv complexity of cortical activity during sleep and waking in rats," Journal of Neurophysiology, vol. 113, no. 7, pp. 2742-2752, 2015.

[68] M. W. Rivolta, M. Migliorini, M. Aktaruzzaman, R. Sassi, and A. M. Bianchi, "Effects of the series length on Lempel-Ziv Complexity during sleep," in Proceedings of the 2014 36th Annual International Conference of the IEEE Engineering in Medicine and Biology Society, EMBC 2014, pp. 693-696, USA, August 2014.

[69] X.-S. Zhang, R. J. Roy, and E. W. Jensen, "EEG complexity as a measure of depth of anesthesia for patients," IEEE Transactions on Biomedical Engineering, vol. 48, no. 12, pp. 1424-1433, 2001.

[70] M. Aboy, R. Hornero, D. Abásolo, and D. Álvarez, "Interpretation of the Lempel-Ziv complexity measure in the context of biomedical signal analysis," IEEE Transactions on Biomedical Engineering, vol. 53, no. 11, pp. 2282-2288, 2006.

[71] S. Simons and D. Abásolo, "Distance-based Lempel-Ziv complexity for the analysis of electroencephalograms in patients with Alzheimer's disease," Entropy, vol. 19, no. 3, Article ID e19030129, 2017.

[72] R. Hornero, D. Abásolo, J. Escudero, and C. Gómez, "Nonlinear analysis of electroencephalogram and magnetoencephalogram recordings in patients with Alzheimer's disease," Philosophical Transactions of the Royal Society A: Mathematical, Physical \& Engineering Sciences, vol. 367, no. 1887, pp. 317-336, 2009.

[73] R. Hornero, J. Escudero, A. Fernández, J. Poza, and C. Gómez, "Spectral and nonlinear analyses of MEG background activity in patients with Alzheimer's disease," IEEE Transactions on Biomedical Engineering, vol. 55, no. 6, pp. 1658-1665, 2008.

[74] A. Fernandez, R. Hornero, C. Gómez et al., "Complexity analysis of spontaneous brain activity in alzheimer disease and mild cognitive impairment: An MEG study," Alzheimer Disease \& Associated Disorders, vol. 24, no. 2, pp. 182-189, 2010.

[75] A. Accardo, M. Affinito, M. Carrozzi, and F. Bouquet, "Use of the fractal dimension for the analysis of electroencephalographic time series," Biological Cybernetics, vol. 77, no. 5, pp. 339-350, 1997.

[76] H. Preißl, W. Lutzenberger, F. Pulvermüller, and N. Birbaumer, "Fractal dimensions of short EEG time series in humans," Neuroscience Letters, vol. 225, no. 2, pp. 77-80, 1997.

[77] T. Higuchi, "Approach to an irregular time series on the basis of the fractal theory," Physica D: Nonlinear Phenomena, vol. 31, no. 2, pp. 277-283, 1988.

[78] R. Esteller, G. Vachtsevanos, J. Echauz, and B. Litt, "A comparison of fractal dimension algorithms using synthetic and 
experimental data," in Proceedings of the IEEE International Symosium on Circuits and Systems (ISCAS '99), vol. 3, pp. 199202, IEEE, Orlando, Fla, USA, July 1999.

[79] C. Gómez, Á. Mediavilla, R. Hornero, D. Abásolo, and A. Fernández, "Use of the Higuchi's fractal dimension for the analysis of MEG recordings from Alzheimer's disease patients," Medical Engineering \& Physics, vol. 31, no. 3, pp. 306-313, 2009.

[80] F. M. Smits, C. Porcaro, C. Cottone, A. Cancelli, P. M. Rossini, and F. Tecchio, "Electroencephalographic fractal dimension in healthy ageing and Alzheimer's disease," PLoS ONE, vol. 11, no. 2, Article ID e0149587, 2016.

[81] T. Staudinger and R. Polikar, "Analysis of complexity based EEG features for the diagnosis of Alzheimer's disease," in Proceedings of the 33rd Annual International Conference of the IEEE Engineering in Medicine and Biology Society, EMBS 2011, pp. 2033-2036, USA, September 2011.

[82] L. Hirsch and R. Brenner, Atlas of EEG in critical care, John Wiley \& Sons, 2011.

[83] S. Sanei, Adaptive processing of brain signals, John Wiley \& Sons, 2013.

[84] M. F. Folstein, S. E. Folstein, and P. R. McHugh, “"'Mini mental state". A practical method for grading the cognitive state of patients for the clinician," Journal of Psychiatric Research, vol. 12, no. 3, pp. 189-198, 1975.

[85] E. Strauss, E. M. S. Sherman, and O. Spreen, A compendium of neuropsychological tests: Administration, norms, and commentary, American Chemical Society, 2006.

[86] A. L. Benton, Revised Visual Retention Test: Clinical and Experimental Applications, Psychological Corporation, New York, NY, USA, 1974.

[87] G. A. Talland and M. Ekdahl, "Psychological studies of korsakoff's psychosis: IV. The rate and mode of forgetting narrative material," The Journal of Nervous and Mental Disease, vol. 129, no. 4, pp. 391-404, 1959.

[88] J. Tuqan and P. P. Vaidyanathan, "Optimum low cost two channel IIR orthonormal filter bank," in Proceedings of the 1997 IEEE International Conference on Acoustics, Speech, and Signal Processing, ICASSP, pp. 2425-2428, April 1997.

[89] M. Gell-Mann and C. Tsallis, Nonextensive entropy: interdisciplinary applications, Oxford University Press, 2004.

[90] C. Gómez, D. Abásolo, J. Poza, A. Fernández, and R. Hornero, "MEG analysis in Alzheimer's disease computing approximate entropy for different frequency bands," in Proceedings of the 2010 32nd Annual International Conference of the IEEE Engineering in Medicine and Biology Society, EMBC'10, pp. 2379-2382, Argentina, September 2010.

[91] J. Ramírez, J. M. Górriz, D. Salas-Gonzalez et al., "Computeraided diagnosis of Alzheimer's type dementia combining support vector machines and discriminant set of features," Information Sciences, vol. 237, pp. 59-72, 2013.

[92] R. Chaves, J. Ramírez, J. M. Górriz et al., "SVM-based computer-aided diagnosis of the Alzheimer's disease using ttest NMSE feature selection with feature correlation weighting," Neuroscience Letters, vol. 461, no. 3, pp. 293-297, 2009.

[93] J. M. Górriz, J. Ramírez, A. Lassl et al., "Automatic computer aided diagnosis tool using component-based SVM," in Proceedings of the 2008 IEEE Nuclear Science Symposium Conference Record, NSS/MIC 2008, pp. 4392-4395, Germany, October 2008.

[94] A. So, D. Hooshyar, K. W. Park, and H. S. Lim, "Early diagnosis of dementia from clinical data by machine learning techniques," Applied Sciences (Switzerland), vol. 7, no. 7, article no. 651, 2017.
[95] S. Raschka, An Overview of General Performance Metrics of Binary Classifier Systems, 2014, arXiv1410.5330.

[96] D. M. W. Powers, "Evaluation: From Precision, Recall and F-Factor to ROC, Informedness, Markedness \& Correlation, School of Informatics and Engineering, Flinders University, Adelaide, Australia," Journal of Machine Learning Technologies, vol. 2, no. 1, pp. 37-63, 2011.

[97] S. Simons, D. Abasolo, and M. Hughes, "Investigation of Alzheimer's disease EEG frequency components with LempelZiv complexity," in Proceedings of the 6th European Conference of the International Federation for Medical and Biological Engineering, MBEC 2014, pp. 46-49, Croatia, September 2014.

[98] N. N. Kulkarni and V. K. Bairagi, "Extracting Salient Features for EEG-based Diagnosis of Alzheimer's Disease Using Support Vector Machine Classifier," IETE Journal of Research, vol. 63, no. 1, pp. 11-22, 2017.

[99] D. V. Moretti, “Theta and alpha eeg frequency interplay in subjects with mild cognitive impairment: Evidence from EEG, MRI and spect brain modifications," Frontiers in Aging Neuroscience, vol. 7, article no. 31, 2015.

[100] C. Babiloni, R. Ferri, G. Binetti et al., "Directionality of EEG synchronization in Alzheimer's disease subjects," Neurobiology of Aging, vol. 30, no. 1, pp. 93-102, 2009.

[101] U. A. Khan, L. Liu, F. A. Provenzano et al., "Molecular drivers and cortical spread of lateral entorhinal cortex dysfunction in preclinical Alzheimer's disease," Nature Neuroscience, vol. 17, no. 2, pp. 304-311, 2014. 


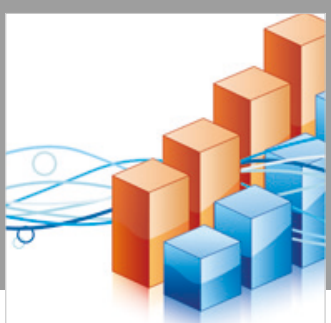

Advances in

Operations Research

\section{-n-m}
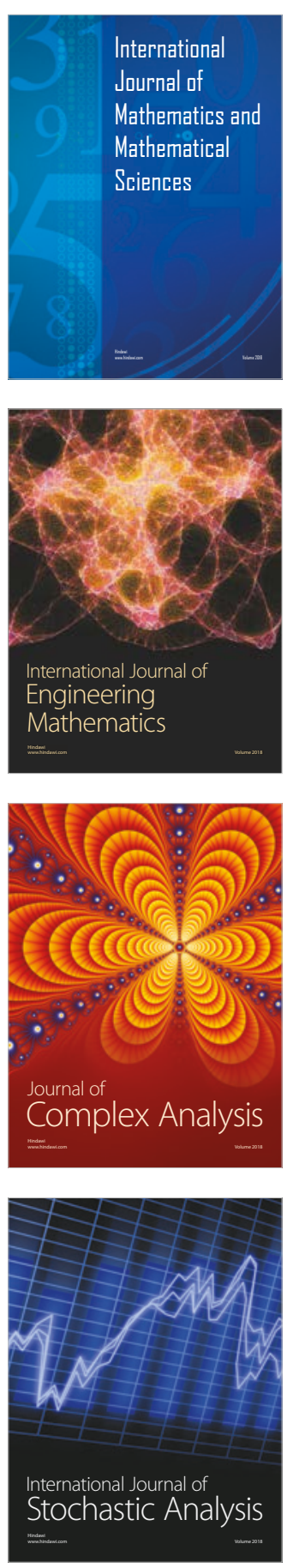
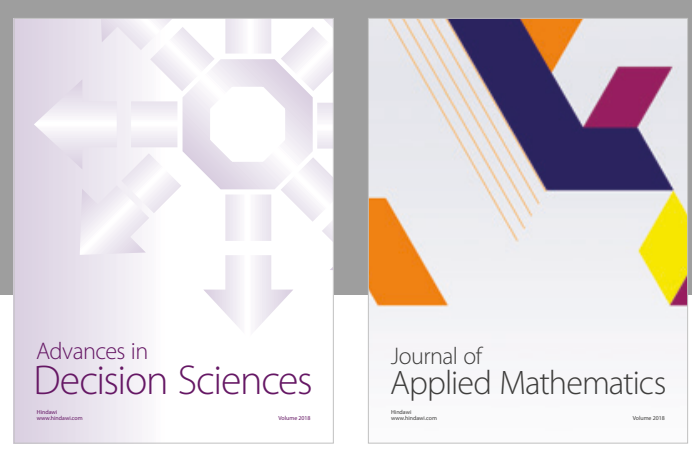

Journal of

Applied Mathematics
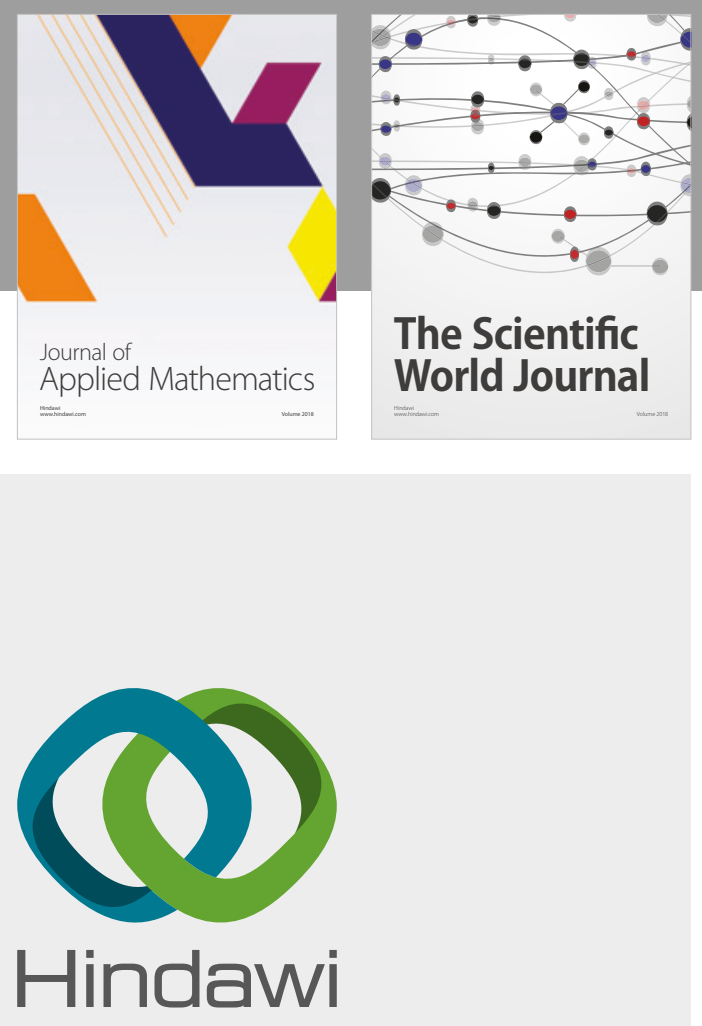

Submit your manuscripts at

www.hindawi.com

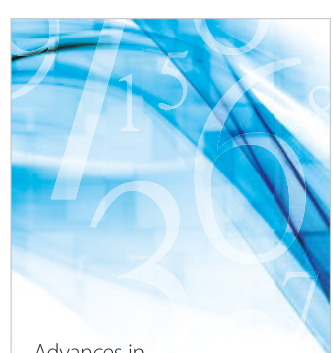

Advances in
Numerical Analysis
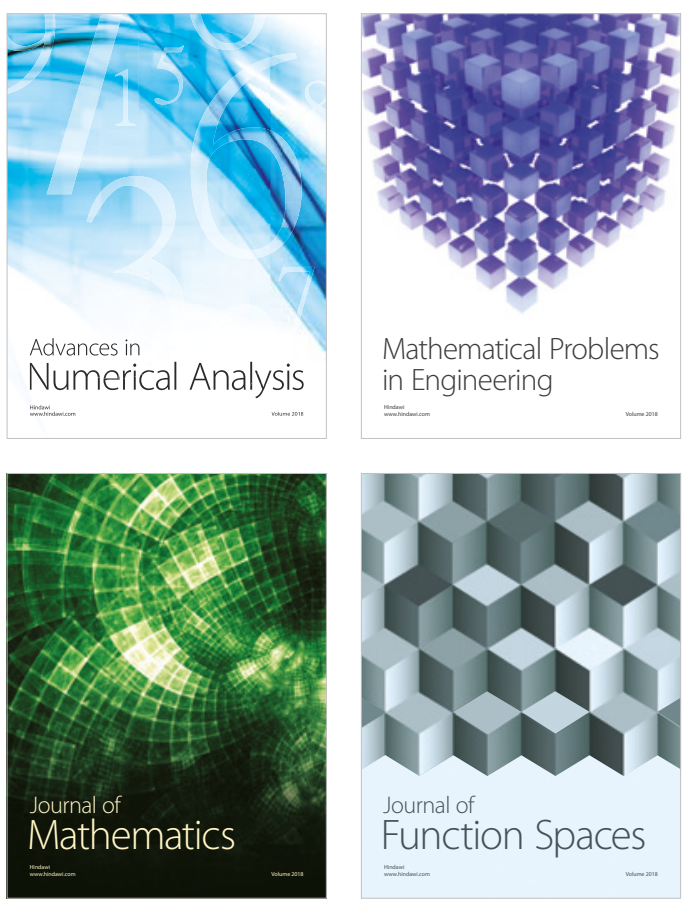

Mathematical Problems in Engineering

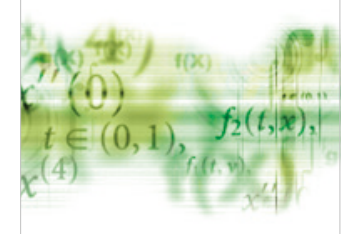

International Journal of

Differential Equations

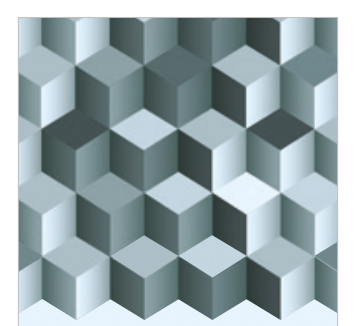

Journal of

Function Spaces
The Scientific

World Journal

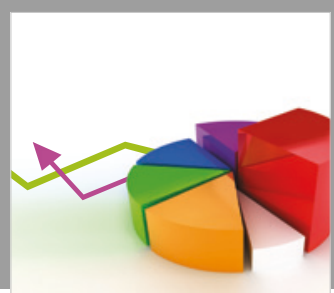

Journal of

Probability and Statistics
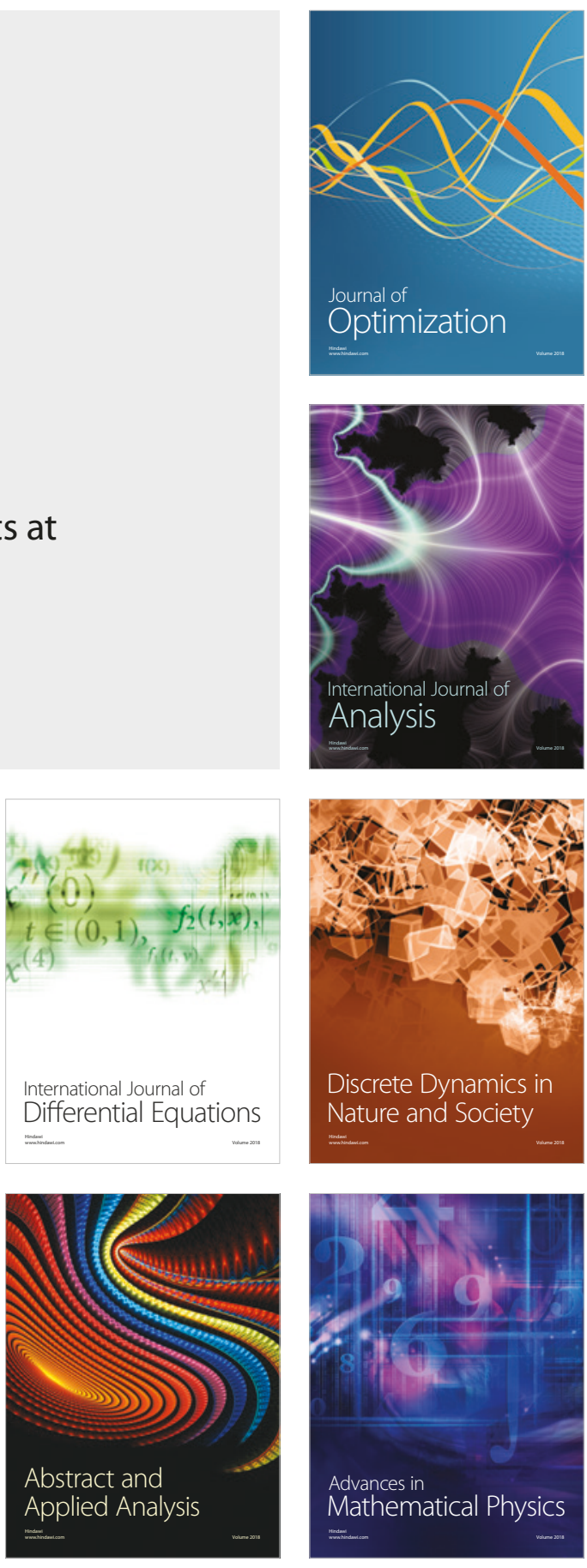